\title{
BMJ Open Preoperative contingency management intervention for smoking abstinence in cancer patients: trial protocol for a multisite randomised controlled trial
}

\author{
Alana M Rojewski (D) , ${ }^{1,2}$ Lisa M Fucito, ${ }^{3}$ Nathaniel L Baker, ${ }^{1}$ Amanda M Palmer,,${ }^{1,4}$ \\ Madeline G Foster, ${ }^{1}$ Graham W Warren, ${ }^{2,5}$ Steven L Bernstein, $, 6,7,8$ \\ Benjamin A Toll ${ }^{1,2,9}$
}

To cite: Rojewski AM, Fucito LM, Baker NL, et al. Preoperative contingency management intervention for smoking abstinence in cancer patients: trial protocol for a multisite randomised controlled trial. BMJ Open 2021;11:e051226. doi:10.1136/ bmjopen-2021-051226

- Prepublication history for this paper is available online. To view these files, please visit the journal online (http://dx.doi. org/10.1136/bmjopen-2021 051226).

Received 16 March 2021 Accepted 03 June 2021

Check for updates

(C) Author(s) (or their employer(s)) 2021. Re-use permitted under CC BY-NC. No commercial re-use. See rights and permissions. Published by BMJ.

For numbered affiliations see end of article.

Correspondence to

Dr Alana M Rojewski;

rojewski@musc.edu

\section{ABSTRACT}

Introduction Continued smoking following a cancer diagnosis has substantial health risks including increased overall and cancer-specific mortality, risk of secondary malignancies, cancer treatment toxicity and risk of surgical complications. These risks can be mitigated by quitting smoking. The preoperative period represents a prime opportunity in which to administer robust smoking cessation treatment to both improve health and support and improve surgical outcomes. We will conduct a randomised clinical trial to evaluate the effectiveness of financial incentives delivered contingent on biochemically verified smoking abstinence (contingency management (CM)) in patients with cancer undergoing surgery.

Methods and analysis The study will take place across two study sites, and participants ( $\mathrm{N}=282)$ who smoke, are diagnosed with or suspected to have any type of operable cancer and have a surgical procedure scheduled in the next 10 days to 5 weeks will be randomised to receive standard care plus Monitoring Only or CM prior to surgery. All patients will receive breath carbon monoxide (CO) tests three times per week, nicotine replacement therapy and counselling. The CM group will also earn payments for self-reported smoking abstinence confirmed by $\mathrm{CO}$ breath test $\leq 4 \mathrm{ppm}$ on an escalating schedule of reinforcement (with a reset if they smoked). Point prevalence abstinence (PPA) outcomes (self-report of 7-day abstinence confirmed by $\mathrm{C} 0 \leq 4 \mathrm{ppm}$ and/or anabasine $\leq 2 \mathrm{ng} / \mathrm{mL}$ ) will be assessed on the day of surgery and 6 months after surgery. The effect of CM on 7-day PPA at the time of surgery and 6-month follow-up will be modelled using generalised linear mixed effects models.

Ethics and dissemination This study has been reviewed and approved by the Medical University of South Carolina Institutional Review Board. We will disseminate our scientific results through traditional research-oriented outlets such as presentations at scientific meetings and publications in peer-reviewed journals.

Trial registration number NCT04605458.

\section{INTRODUCTION}

Tobacco use among patients with cancer is high, ${ }^{1}$ with some estimates as high as $18 \%$ overall and up to $27 \%$ of tobacco-related
Strengths and limitations of this study

- This is the first fully powered, large-scale smoking cessation clinical trial to test a contingency management intervention for patients with cancer undergoing surgery.

- Exploratory aim includes an evaluation of the effect of smoking abstinence on postsurgical outcomes (ie, wound healing and respiratory complications), which will be the first evaluation of how smoking abstinence achieved through an incentive-based behavioural intervention can affect postsurgical outcomes in patients with cancer.

- The abstinence outcomes will be verified by breath carbon monoxide and/or urine anabasine to ensure optimal biochemical verification of abstinence.

- The three-times-per-week breath sampling schedule may allow for smoking to go undetected for brief periods of time during the intervention phase.

patients with cancer. ${ }^{2}$ Continued smoking after initial diagnosis can increase both overall and cancer-specific mortality, ${ }^{3}$ increase the likelihood of developing secondary malignancies $^{4-6}$ and negatively impact response to all forms of treatment (surgery, radiation, chemotherapy). ${ }^{7-11}$ For patients who are undergoing treatment for cancer, especially at earlier stages, surgery is often the most common treatment choice. ${ }^{12}$ All smokers undergoing surgery for cancer (regardless of site) are at an increased risk of postoperative complications, such as infections and respiratory complications. ${ }^{7913}$ Smoking after a diagnosis of cancer contributes to numerous preventable problems, and it is critical for patients to quit.

Quitting smoking reduces many of the above referenced adverse effects, with one study demonstrating better 5-year survival of former smokers and recent quitters 
compared with current smokers. ${ }^{14}$ Smoking cessation after diagnosis also reduces cancer recurrence or cancerspecific mortality ${ }^{15} 16$ and decreases overall complications after surgery. ${ }^{17}$ The timing of smoking cessation as related to surgery is also important: in 1330 patients with gastric cancer undergoing surgery for radical gastrectomy, smoking abstinence for a period of at least 2 weeks was associated with a reduction in complication rates. ${ }^{17}$ Unfortunately, many patients with cancer are unable to quit smoking following their diagnosis. ${ }^{18} 19$

Relatively few tobacco treatment interventions for patients with cancer surgery have been investigated, and none have shown large differences between intervention and control groups. To date, only two studies have prospectively tested interventions for tobacco cessation before cancer surgery, one evaluating motivational interviewing and one evaluating a scheduled reduced smoking intervention. ${ }^{20}{ }^{21}$ However, neither of these studies examined financial incentives or demonstrated a benefit for the intervention group, and any abstinence rates that were achieved were not sustained at follow-up. Achieving abstinence prior to surgery and abstinence maintenance after surgery is a critical component to the treatment of patients with surgical cancer in order to improve initial surgical outcomes as well as reduce long-term morbidity and mortality.

Behaviours such as cigarette smoking are maintained in part by the reinforcing effects of smoking and environmental stimuli. ${ }^{22}$ Thus, smoking behaviour can be altered through the manipulation of behavioural contingencies; that is, reinforcing and/or punishing consequences of smoking. ${ }^{22-24}$ Contingency management $(\mathrm{CM})$ is a treatment strategy within this conceptual framework that uses reinforcing consequences (ie, reward incentives) when patients achieve the target behaviour (ie, abstain from smoking) and withholds reinforcement when patients do not. ${ }^{22}$ There is a large body of research showing that $\mathrm{CM}$ promotes reduction, abstinence and long-term abstinence from tobacco. ${ }^{25-27}$ This treatment approach has been used successfully to reduce the amount of smoking in both treatment seeking ${ }^{28}$ and nontreatment seeking smokers ${ }^{29}$ and has consistently produced high quit rates among high-risk smokers. ${ }^{30-35}$

Initial pilot data suggest that CM may also be a promising intervention in presurgical patients with cancer who smoke. Our prior work calculated effect size estimates for a presurgical CM intervention and showed that delivering financial incentives contingent on biochemically confirmed abstinence from tobacco resulted in a $36 \%$ higher abstinence rate the week before surgery compared with a group who received no incentives for biochemical monitoring of smoking status $(52 \%$ vs $16 \%$, respectively, $\mathrm{p}=0.03) .{ }^{36}$ Importantly, this $36 \%$ difference showed durability at the 3-month follow-up ( $43 \%$ vs $5 \%, \mathrm{p}=0.02$ ). It is noteworthy that even though the active control condition included evidence-based tobacco treatment (ie, counselling + nicotine replacement therapy (NRT)), the CM intervention still showed a $36 \%$ higher quit rate. These preliminary results suggest that adding financial incentives for abstinence to evidence-based tobacco treatment may improve abstinence rates for presurgical patients with cancer.

This study will evaluate the effectiveness of a CM intervention on smoking abstinence in a multisite, full-powered randomised trial of patients with cancer undergoing surgery. The primary outcome for this trial is biochemically confirmed abstinence on the day of surgery.

\section{METHODS AND ANALYSIS}

This clinical trial protocol follows the Standard Protocol Items: Recommendations for Interventional Trials guidelines $^{37}$ and is registered on clinicaltrials.gov. Study enrolment began on 25 November 2020, and the estimated primary completion date is 15 December 2024.

\section{Participants}

The target sample will be 282 preoperative patients with cancer who smoke across two study sites: Hollings Cancer Center at the Medical University of South Carolina (HCC) and Yale Cancer Center/Smilow Cancer Hospital at Yale-New Haven (YCC). Inclusion criteria include: (1) age $\geq 18$ years, (2) smoking $\geq 1$ cigarettes per day (CPD), confirmed by breath carbon monoxide $(\mathrm{CO})>4$ parts per million (ppm), (3) diagnosed with or suspicion of any type of operable cancer and scheduled for surgery. Exclusion criteria include: (1) unstable psychiatric/medical conditions, (2) non-English speaking, (3) use of alternative nicotine delivery systems (eg, NRT, e-cigarettes etc) and (4) pregnant women.

\section{Screening}

A research assistant (RA) will monitor provider clinic schedules via the electronic medical record, and proactively and retroactively screen for potential participants through chart review. The RA will contact a potential participant's oncology care team to attend the clinic appointment and inform the patient about the study. The care team members may also provide referrals to the RA. Patients who express interest in the study will meet with an RA in person (YCC or HCC) or remotely for an intake appointment.

\section{Consent}

Participants will have the option to complete consent and/or the baseline session (1) written, in person, (2) electronically, through a Health Insurance Portability and Accountability Act (HIPAA)-compliant secure virtual platform (eg, REDCap, Doxy.me etc), (3) written, by postal mail combined with a phone discussion or (4) a combination of these options.

\section{Intake session}

Study eligibility will be determined in concert with the treating physician and the principal investigator (PI). If the patient consents to participate, they will be enrolled and will complete the intake session. Medical and tobacco 
use histories, breath CO levels and pregnancy tests (if applicable) will be obtained from all participants using a combination of in-person and remote visits for data collection.

After the intake session, the RA will schedule the week's breath monitoring sessions and the weekly counselling sessions based on the participant's scheduled surgery (10 days to 5 weeks from the intake). The RA will also administer the first week of NRT either in person or through mail.

\section{Randomisation}

At the time of the intake, participants who consent to participate in the study will be randomised by an RA to standard care plus Monitoring Only (MO; counselling, NRT and breath monitoring) or CM (MO plus financial incentives delivered contingent on abstinent breath samples) in a 1:1 allocation. Randomisation will be stratified across (a) site (HCC or YCC) and (b) length of treatment ( $>21$ days or $\leq 21$ days between study initiation and surgery date) using a permuted random block design with varying block sizes $(2,4$ or 6$)$.

\section{Intervention}

All participants (MO and CM groups) will receive counselling, NRT and breath monitoring to verify smoking status as described below. The CM group will also receive incentives contingent on abstinence from smoking. The intervention period will conclude on the day of surgery with a final breath monitoring visit and a urine anabasine test to assess smoking status. ${ }^{38}$ If the date of surgery is changed, the schedule of procedures will be adapted to fit the new presurgical period as best as possible. If the surgery is rescheduled beyond the 5-week intervention, the patient will receive treatment for a maximum of 5 weeks. If surgery is cancelled, the protocol will continue as described until the date of originally scheduled surgery.

\section{Counselling}

All participants will receive 3-6 counselling sessions from a tobacco treatment specialist (nurse practitioner, clinical pharmacist or psychology resident). The counselling protocol will be based on practical counselling, a cognitive behavioural evidence-based smoking cessation treatment modality, ${ }^{39}$ which is the manualised, standardof-care counselling used by the Tobacco Treatment Programme at HCC and the Tobacco Treatment Service at YCC. Participants will receive two counselling sessions in the first 7 days following their intake and will be asked to set a quit date within 1-3 days of the first counselling session. After the first week, participants will receive counselling once per week, ending with surgery (maximum six sessions). If a participant has a 10-day presurgical time period, 3 counselling sesions will be condensed into 10 days before surgery. Skills covered in counselling will include the following as applicable based on participant needs: preparing to quit, medication use, coping with urges and triggers, stress management, managing withdrawal symptoms, behavioural activation and social support. Counselling fidelity will be monitored using an adapted Yale Adherence and Competence Scale ${ }^{40} 41$ to be completed by counselling providers. In addition to the weekly counselling, all participants will be asked to complete a weekly battery of assessments and will be paid US $\$ 5$.

\section{Pharmacotherapy}

Participants will be provided dual NRT (seven patches and three vials of mini lozenges) each week, ending with surgery (up to 5 weeks). Dosing will be consistent with the package insert information for both products. Patches will be provided based on baseline self-reported smoking levels (21 mg for $>10 \mathrm{CPD}$ and $14 \mathrm{mg}$ for $\leq 10 \mathrm{CPD}$ ), and lozenges will be provided based on baseline self-reported time to first cigarette $(4 \mathrm{mg}$ if they smoke within $30 \mathrm{~min}$ of waking and $2 \mathrm{mg}$ if they smoke $>30 \mathrm{~min}$ after waking). Dose adjustments will be allowed based on clinical judgement of the respective site PIs with input from the study tobacco treatment specialists.

\section{Breath CO testing}

All participants will provide breath $\mathrm{CO}$ tests three times per week, with the final $\mathrm{CO}$ test occurring on the day of surgery (minimum breath tests $=5$; maximum $=15$ ). $\mathrm{CO}$ breath tests will be conducted either in person or remotely. All breath tests conducted in person will be done using a Bedfont Micro+Smokerlyzer following the health and safety precautions outlined by Bedfont. All breath tests conducted remotely will be done using a Bedfont iCO Smokerlyzer and a webcam (if needed), which will be mailed to the participant. The RA will contact the patient via a HIPAA compliant video conferencing platform to observe them recording the test to verify patient identity, breath collection procedures and results. Tests will occur on Mondays, Fridays and one additional day in between.

\section{CM intervention}

For those randomised to the CM condition, payment for abstinence will be contingent on self-report of no smoking since the last visit confirmed by breath CO $\leq 4 \mathrm{ppm} .{ }^{38}$ The incentive schedule is shown in table 1 . The incentives start at US $\$ 15$ and increase in US $\$ 5$ increments until participants reach US $\$ 55$ per negative breath test, where the earnings will be capped for all subsequent payments in order to control for magnitude of the intervention across participants. Incentives will be withheld (US\$0) for breath CO $>4 \mathrm{ppm}$ and missing samples, resulting in a reset to US $\$ 15$ for the next negative sample. The second negative test would result in payment equal to the last achieved value if the next sample $\mathrm{CO} \leq 4 \mathrm{ppm}$ (eg, if a participant provided a $\mathrm{CO}$ sample $\leq 4 \mathrm{ppm}$ for CM meeting 3, they would earn US $\$ 25$; a CO > 4 ppm for CM meeting 4 would earn US $\$ 0$; a $\mathrm{CO} \leq 4 \mathrm{ppm}$ for CM meeting 5 would earn US $\$ 15$ and a $\mathrm{CO} \leq 4 \mathrm{ppm}$ for CM meeting 6 would earn US\$25). Participants can earn US\$125 for perfect abstinence 


\begin{tabular}{lcc}
\hline Table 1 & Schedule of reinforcement & \\
\hline Week & Breath test & Payment \\
\hline 1 & 1 & US $\$ 15$ \\
& 2 & US $\$ 20$ \\
& 3 & US $\$ 25$ \\
\hline & 4 & US $\$ 30$ \\
& 5 & US $\$ 35$ \\
& 6 & US $\$ 40$ \\
3 & 7 & US $\$ 45$ \\
& 8 & US $\$ 50$ \\
& 9 & US $\$ 55$ \\
4 & 10 & US $\$ 55$ \\
& 11 & US $\$ 55$ \\
& 12 & US $\$ 55$ \\
5 & 13 & US $\$ 55$ \\
& 14 & US $\$ 55$ \\
& 15 & US $\$ 55$ \\
\hline
\end{tabular}

through 10 days and up to US\$645 if treatment (and abstinence) lasts 5 weeks. Payment will be delivered in the form of an Amazon e-gift card payment or cash immediately delivered to participants on biochemical verification of abstinence. The MO group will receive no financial incentives for breath tests.

We will implement treatment fidelity procedures to ensure consistent delivery of the experimental CM intervention and assessments. ${ }^{42-44}$ This includes: (1) all staff will initially be trained and supervised by the site PI in delivery of CM with a focus on delivery of contingent payments following the prespecified schedule of reinforcement, (2) after live training, including observed contingent payments with both mock and real research participants, weekly auditing of the payment forms to ensure that participants are seen 3 days per week and paid the amount they were scheduled to be paid at the time of $\mathrm{CO}$ breath test, (3) periodic review and refresher training to prevent drift in protocol implementation and to ensure consistency within and across sites, (4) ongoing monitoring and documentation of unexpected protocol deviations.

\section{Follow-up}

Participants will complete assessments and a breath CO test at 3-month and 6-month follow-up sessions. The participant will also provide a urine sample for anabasine testing at the 6-month follow-up. These assessments will be completed in person, by phone or through REDCap's automated survey feature. Surveys will automatically be sent to participants at the time of their 3-month and 6-month follow-up. We will pay participants US $\$ 25$ for completion of the 3-month and 6-month follow-up interviews.

\section{Measures}

Participants will complete a series of baseline measures at their first meeting in person, by phone or through REDCap's automated survey feature, including demographic and smoking history questionnaires. The Fagerström Test for Nicotine Dependence will be completed at baseline to assess the severity of dependence on nicotine and to tailor the dosing for the nicotine lozenge using the time-to-first-cigarette item. ${ }^{45-47}$ Quantity and frequency estimates of tobacco, alcohol and NRT use will be assessed using a Timeline Follow-Back Procedure starting at 30 days prior to randomisation (repeated weekly, at the end of treatment and at follow-ups). ${ }^{48-50}$ The Systematic Assessment for Treatment Emergent Events will be completed at baseline and weekly throughout the trial to assist with identifying any treatment-related adverse events. ${ }^{51}$ Participants will also complete a Medication Adherence Questionnaire to assess self-reported ability to adhere to medication regimens (repeated weekly and at follow-ups),$^{52}$ the Snaith-Hamilton Pleasure Scale to assess the pleasure derived from naturalistic reinforcers (modified; repeated at end of treatment and follow-ups), ${ }^{53}$ the modified Cigarette Evaluation Questionnaire to assesses the degree to which participants experience the reinforcing effects of smoking (repeated at the end of treatment and follow-ups), ${ }^{54}$ the Patient Health Questionnaire-4 to measures symptoms of depression and anxiety (repeated weekly and at the end of treatment), ${ }^{55}$ self-efficacy as assessed by self-reported confidence to quit, ${ }^{56}$ motivation and confidence to quit smoking using the Contemplation Ladder, ${ }^{57}$ outcome expectancies as assessed by the degree to which they expect that quitting smoking will impact their health down the road and quality of life as assessed by the WHO Quality of LifeBrief Version (5-item, modified; repeated at follow-ups).$^{59}$ Medical and surgical outcomes will be recorded based on chart review during admission and in the 30 days after surgery. $^{79}$

\section{Outcomes}

The primary outcome will be 7-day point prevalence abstinence (PPA) defined as no cigarettes (or other tobacco products) for 7 days by self-report, confirmed by breath $\mathrm{CO} \leq 4 \mathrm{ppm}$ and/or urine anabasine levels $\leq 2 \mathrm{ng} / \mathrm{mL}$ on the day of surgery. If the anabasine is lost or unusable, or if the reason we were unable to obtain the urine sample was no fault of the participant, then we will default to $\mathrm{CO}$ only. The sample will be coded as positive for smoking if (1) the participant refuses the urine sample, (2) the CO is negative and the anabasine is positive or (3) the $\mathrm{CO}$ is positive and the anabasine is negative. For secondary outcomes, 7-day PPA will also be assessed at the 3-month and 6-month follow-ups.

\section{Power and sample size}

We will test the primary hypothesis that rates of smoking abstinence will be significantly higher at the time of surgery in the CM group as compared with the MO 
group. In our previous pilot trial $(n=40), 52 \%(11 / 21)$ of those who received CM were abstinent on the day of surgery compared with $16 \%(3 / 19)$ of the patients in $\mathrm{MO}$ $\left(\Delta=36.6 \%\right.$; $\mathrm{s}_{\Delta}=13.3 \%$; adjusted RR (95\% CI) 3.3 (1.1 to $9.7)$ ). It has been noted that parameter estimates from pilot trials tend to provide overly optimistic CIs around resulting estimates. ${ }^{60} 61$ Thus, we will assume a more conservative biologically confirmed 7-day PPA rate at the time of surgery of $20 \%$ in the MO group and an abstinence rate of $40 \%$ in the CM group $(\Delta=20 \%)$. Based on these estimates, we will have $80 \%$ power with a type 1 error rate of $5 \%$ to detect the difference between those receiving CM (40\%) and those receiving MO (20\%) with 82 participants randomised to each of the two treatment groups (total $n=164$ ). Randomisation will be done at the participant level and stratified by site and treatment length, thus we expect very modest between site variation as compared with the total variance $(\sim \rho=0.01)$; however, due to sampling of participants across two distinct sites, we will inflate the necessary sample size to $n=141$ participants per group to account for this possible loss of power. This results in a total of $\mathrm{N}=282$ randomised participants across both sites to maintain adequate power to detect the clinically meaningful difference stated.

This proposed study size will also provide sufficient power to detect important difference in abstinence rates at the 3-month and 6-month follow-up visits between groups. In the pilot study, those who received the preoperative CM intervention for smoking abstinence, $43 \%$ $(9 / 21)$ were abstinent at the 3-month follow-up visit compared with $5 \%(1 / 19)$ of the patients in the MO group $\left(\Delta=37.6 \% ; \mathrm{s}_{\Delta}=12.0 \%\right.$; adjusted RR $(95 \% \mathrm{CI}) 8.6$ (1.5 to 49.4)). Similar to hypothesis 1 , we assume a conservative biologically confirmed 7-day PPA rate at the 3-month and 6-month follow-up visit of $10 \%$ based on pilot response data and U.S. Public Health Service guidelines for the participants randomised to receive $\mathrm{MO}$ and an abstinence rate of $30 \%$ in the CM group. Accounting for a betweenvisit (two follow-up visits), within-participant correlation of $\rho=0.95$ and an attrition rate between surgery and the follow-up visits of $20 \%$, the randomised sample of 282 participants will provide greater than $80 \%$ power to detect the stated difference in abstinence rates at the 3 -month and 6 month follow-ups. All comparisons are powered using a two-sided type 1 error rate of $5 \%$ and no correction for multiple comparisons is applied to sample size estimates.

\section{Planned analyses}

Data will be collected on a secure, password-protected, electronic web-based form and sent to a secure database in REDCap. Only research staff have access to the database. Participants will be assigned a study ID to protect confidentiality. The PI will be responsible for monitoring the data, assuring protocol compliance and conducting the safety reviews.

Clinical and demographic variables will be tabulated across treatment groups. Preliminary analysis of the association of baseline clinical, demographic and biological characteristics with biologically confirmed abstinence at each study milestone (surgery, 3-month and 6 month follow-up) will be examined in the study population using logistic regression models. Characteristics found to be significantly associated with primary study outcomes will be included as covariates in the initial stages of adjusted model development.

The effect of CM on 7-day PPA at the time surgery and 3-month and 6 month follow-up visits will be modelled using generalised linear mixed effects models with $\log$ link function and a Poisson distribution to estimate relative risks. Primary (surgery) and secondary (3-month and 6 month follow-up) design-adjusted outcome models will be assessed with the inclusion of randomised treatment assignment, baseline smoking characteristics, the type of cancer (tobacco-related cancer vs other), adjuvant cancer treatment prior to surgery (radiation and/or chemotherapy or none), length of treatment (ie, days until surgery), study site and visit (where appropriate). Additional covariate-adjusted models will contain significant covariates found in the preliminary analysis. For these efficacy analyses, 7-day PPA will be defined as stated above in the Outcomes section. All randomised participants will be included in the analyses (intent-to-treat (ITT) approach) ${ }^{62}$ and participants will be considered nonabstinent at any missed visit (drop-out/lost-to-follow-up included). In addition to the primary ITT analyses, exploratory approaches (eg, modified ITT models, perprotocol analysis, completer analysis and multiple imputation methods) will be undertaken as indicated and a sensitivity analysis comparing these models to the ITT model will be completed. Methods of multiple imputation will be implemented using fully conditional specification with a logistic regression modelling approach. ${ }^{63}$ One hundred imputation data files will be created to assure reasonable relative efficiency ${ }^{64}$ Imputation of biologically confirmed smoking status at the end of treatment and follow-up will be based on model variables (randomised treatment assignment, baseline-reported CPD, years of regular smoking, sex, cancer type, treatment length and participant age). Imputed values for smoking status will be included in a logistic regression model and combined to generate parameter estimates and 95\% CIs. Risk ratios (RRs) and asymptotic 95\% CIs will be computed for efficacy estimates.

An exploratory aim to examine the rates of postoperative complications (eg, delayed wound healing) will also be assessed. Complications will be identified in the medical record using a standard form we developed in consultation with surgeons in our preliminary studies. All cause surgical/recovery emergent event frequencies will be compared through the use of generalised linear mixed effect models for count data (Poisson/Negative Binomial). We will also examine exploratory moderators of treatment to determine which subgroups are most likely to respond to the $\mathrm{CM}$ intervention. Variables that predict the outcome variable (abstinence from smoking) 
differently between treatments will be considered to be moderators using the model outlined by Kraemer et al. ${ }^{65}$ We hypothesise that length of treatment, cancer site ${ }^{3}$ and presence of adjuvant cancer treatment before surgery will modify treatment response. Variables that are associated with study outcomes but do not significantly interact with treatment condition will be classified as nonspecific prerandomisation covariates in adjusted analysis. In addition to clinical moderators of treatment efficacy, participant sex will be investigated for effect modification at all time points. ${ }^{66-68}$

Finally, we will examine whether the CM intervention is cost-effective. CM's economic benefit will be calculated as (healthcare costs 30 days postsurgery ${ }_{\mathrm{MO}}$-healthcare costs 30 days postsurgery ${ }_{\mathrm{CM}}$ )-CM cost. We will calculate the cost of $\mathrm{CM}$ as the sum of incentives and the labour costs required to administer the intervention. Postoperative healthcare costs of the CM and MO groups will be obtained from participants' hospital claims records. If $\mathrm{CM}$ results in positive clinical outcomes but not net cost savings, we will estimate the incremental cost-effectiveness ratio of $\mathrm{CM}$ relative to $\mathrm{MO}$ in terms of cost per complication index score reduced (ie, $\left(\operatorname{cost}_{\mathrm{CM}}-\operatorname{cost}_{\mathrm{MO}}\right) /($ complication index score $_{\mathrm{MO}}-$ complication index score $_{\mathrm{CM}}$ ), using the gold panel method). ${ }^{69}$ We will also conduct a scenario analysis to estimate the minimum difference in complication rates between $\mathrm{CM}$ and $\mathrm{MO}$ to justify the cost of CM. Probabilistic sensitivity analyses using Monte Carlo microsimulations will be conducted to test the robustness of our results within reasonable ranges of costs and CM effectiveness. An acceptability curve will be presented to illustrate the probability of CM's cost-effectiveness at different levels of willingness to pay. All statistical analyses will be performed using SAS, V.9.4. ${ }^{70}$

\section{Patient involvement}

Our intervention development began in 2012 with a survey of presurgical cancer patients to ascertain their preferences for abstinence incentives. With each iteration of the intervention, we elicited feedback from participants in the form of exit surveys at the conclusion of their participation. We have assessed satisfaction with the various components of the intervention and used participant experiences and preferences to guide how we structure patient recruitment, interaction, breath test collection and incentive delivery.

\section{Ethics and dissemination}

This study has been reviewed and approved by the MUSC Institutional Review Board (IRB), which serves as the single IRB of record (Pro00099446, last amendment approved 3 August 2021). Participants will sign informed consent before any other study procedures take place. Participants can terminate any part of participation without penalty. If a participant reports an adverse event that may be due to NRT use, we will work with them to ensure that they are using the medication properly. If it is determined that they have been using the medication properly and are still experiencing adverse events, they will be encouraged to discontinue the use of the NRT.

We will disseminate our scientific results through traditional research-oriented outlets such as presentations at scientific meetings and publications in peer-reviewed journals. After the study data are collected and cleaned and the primary paper is published, study data will be available for research purposes to individuals within the scientific community by request. In addition to contributing to the tobacco treatment literature broadly, we hope that this study will help to inform the literature on tobacco treatment options for patients with cancer undergoing surgery.

\section{Author affiliations}

${ }^{1}$ Public Health Sciences, Medical University of South Carolina, Charleston, South Carolina, USA

${ }^{2}$ Hollings Cancer Center, Charleston, South Carolina, USA

${ }^{3}$ Psychiatry, Yale School of Medicine, New Haven, Connecticut, USA

${ }^{4}$ Pulmonary, Critical Care, Allergy, and Sleep Medicine, Medical University of South Carolina, Charleston, South Carolina, USA

${ }^{5}$ Radiation Oncology, Medical University of South Carolina, Charleston, South

Carolina, USA

${ }^{6}$ Dartmouth-Hitchcock Medical Center, Lebanon, New Hampshire, USA ${ }^{7}$ Emergency Medicine, Yale School of Medicine, New Haven, Connecticut, USA ${ }^{8}$ Geisel School of Medicine at Dartmouth, Hanover, New Hampshire, USA

${ }^{9}$ Psychiatry and Behavioral Sciences, Medical University of South Carolina, Charleston, South Carolina, USA

\section{Twitter Alana M Rojewski @AlanaRojewski}

Contributors AMR: conceptualisation, methodology, writing —original draft, writing — review and editing; LMF: methodology, writing — review and editing; NB: data analysis, writing - original draft, writing — review and editing; AMP: writingreview and editing; MGF: writing — review and editing; GW: methodology, writingreview and editing; SLB: conceptualisation, methodology, writing — review and editing; BT: conceptualisation, funding acquisition, methodology, writing—original draft, writing—review and editing.

Funding This research was supported by NCl grants R21CA181569 (BT), R01CA235697 (BT) and K07CA214839 (AMR), and NIH Institutional Postdoctoral Training Grant NIH-T32-HL144470 (AMP).

Competing interests Dr Toll has consulted to Pfizer for an advisory board on e-cigarettes and testifies as an expert witness on behalf of plaintiffs who filed litigation against the tobacco industry.

Patient and public involvement Patients and/or the public were involved in the design, or conduct, or reporting, or dissemination plans of this research. Refer to the Methods section for further details.

\section{Patient consent for publication Not required.}

Provenance and peer review Not commissioned; peer reviewed for ethical and funding approval prior to submission.

Open access This is an open access article distributed in accordance with the Creative Commons Attribution Non Commercial (CC BY-NC 4.0) license, which permits others to distribute, remix, adapt, build upon this work non-commercially, and license their derivative works on different terms, provided the original work is properly cited, appropriate credit is given, any changes made indicated, and the use is non-commercial. See: http://creativecommons.org/licenses/by-nc/4.0/.

\section{ORCID iD}

Alana M Rojewski http://orcid.org/0000-0003-1531-9287

\section{REFERENCES}

1 Toll B, Brandon T, Gritz E. Assessing and stopping cancer patients' Tobacco use: an American Association for Cancer Research (AACR) policy statement. Clinical Cancer Research 2013;19:1941-8. 
2 Day AT, Tang L, Karam-Hage M, et al. Tobacco treatment programs at National cancer Institute-designated cancer centers: a systematic review and online audit. Am J Clin Oncol 2019;42:407-10.

3 US Surgeon General. The health consequences of smoking-50 years of progress: a report of the surgeon General. Atlanta (GA): US Surgeon General, 2014.

4 Kawaguchi T, Matsumura A, luchi K, et al. Second primary cancers in patients with stage III non-small cell lung cancer successfully treated with chemo-radiotherapy. Jpn J Clin Oncol 2006;36:7-11.

5 Do K-A, Johnson MM, Lee JJ, et al. Longitudinal study of smoking patterns in relation to the development of smoking-related secondary primary tumors in patients with upper aerodigestive tract malignancies. Cancer 2004;101:2837-42.

6 Warren GW, Kasza KA, Reid ME, et al. Smoking at diagnosis and survival in cancer patients. Int J Cancer 2013;132:401-10.

7 Sørensen LT, Hørby J, Friis E, et al. Smoking as a risk factor for wound healing and infection in breast cancer surgery. Eur J Surg Oncol 2002;28:815-20.

8 Browman GP, Wong G, Hodson I, et al. Influence of cigarette smoking on the efficacy of radiation therapy in head and neck cancer. N Engl J Med 1993;328:159-63.

9 Myles PS, lacono GA, Hunt JO, et al. Risk of respiratory complications and wound infection in patients undergoing ambulatory surgery: smokers versus nonsmokers. Anesthesiology 2002;97:842-7.

10 Parsons A, Daley A, Begh R, et al. Influence of smoking cessation after diagnosis of early stage lung cancer on prognosis: systematic review of observational studies with meta-analysis. BMJ 2010;340:b5569

11 Mori K, Mostafaei H, Abufaraj M, et al. Smoking and bladder cancer: review of the recent literature. Curr Opin Urol 2020;30:720-5.

12 Miller KD, Siegel RL, Lin CC, et al. Cancer treatment and survivorship statistics, 2016. CA Cancer J Clin 2016;66:271-89.

13 Tellini R, Mari A, Muto G, et al. Impact of smoking habit on perioperative morbidity in patients treated with radical cystectomy for urothelial bladder cancer: a systematic review and meta-analysis. Eur Urol Oncol 2020. doi:10.1016/j.euo.2020.10.006. [Epub ahead of print: 04 Nov 2020].

14 Sardari Nia P, Weyler J, Colpaert C, et al. Prognostic value of smoking status in operated non-small cell lung cancer. Lung Cancer 2005;47:351-9.

15 Passarelli MN, Newcomb PA, Hampton JM, et al. Cigarette smoking before and after breast cancer diagnosis: mortality from breast cancer and smoking-related diseases. J Clin Oncol 2016;34:1315-22.

16 Al-Mamgani A, van Rooij PH, Mehilal R, et al. Radiotherapy for T1a glottic cancer: the influence of smoking cessation and fractionation schedule of radiotherapy. Eur Arch Otorhinolaryngol 2014;271:125-32.

17 Jung KH, Kim SM, Choi MG, et al. Preoperative smoking cessation can reduce postoperative complications in gastric cancer surgery. Gastric Cancer 2015;18:683-90.

18 Cox LS, Africano NL, Tercyak KP. Nicotine dependence treatment for patients with cancer: review and recommendations. Cancer 2003;98:632-44.

19 Blanchard CM, Denniston MM, Baker F, et al. Do adults change their lifestyle behaviors after a cancer diagnosis? Am J Health Behav 2003;27:246-56.

20 Thomsen T, Tønnesen H, Okholm M, et al. Brief smoking cessation intervention in relation to breast cancer surgery: a randomized controlled trial. Nicotine Tob Res 2010;12:1118-24.

21 Ostroff JS, Burkhalter JE, Cinciripini PM, et al. Randomized trial of a presurgical scheduled reduced smoking intervention for patients newly diagnosed with cancer. Health Psychol 2014;33:737-47.

22 Higgins ST, Petry NM. Contingency management. incentives for sobriety. Alcohol Res Health 1999;23:122-7.

23 Higgins ST, Washio Y, Heil SH, et al. Financial incentives for smoking cessation among pregnant and newly postpartum women. Prev Med 2012;55:S33-40.

24 Streck JM, Ochalek TA, Miller ME, et al. Promoting smoking abstinence among patients with chronic obstructive pulmonary disease: initial feasibility. Prev Med Rep 2018;11:176-9.

25 Skinner B. Science and human behavior. New York, NY: Macmillan, 1953.

26 Bigelow G, Silverman K. Theoretical and empirical foundations of contingency management treatments for drug abuse. Washington, DC: American Psychological Association, 1999.

27 Higgins S. Applying learning and conditioning theory to the treatment of alcohol and cocaine abuse. Philadelphia: Lippincott, Williams \& Wilkins, 1997.
28 Secades-Villa R, García-Rodríguez O, López-Núñez C, et al. Contingency management for smoking cessation among treatmentseeking patients in a community setting. Drug Alcohol Depend 2014;140:63-8.

29 Roll JM, Higgins ST. A within-subject comparison of three different schedules of reinforcement of drug abstinence using cigarette smoking as an exemplar. Drug Alcohol Depend 2000;58:103-9.

30 Volpp KG, Troxel AB, Pauly MV, Glick HA, et al. A randomized, controlled trial of financial incentives for smoking cessation. $N$ Engl J Med Overseas Ed 2009;360:699-709.

31 Stitzer ML, Bigelow GE. Contingent reinforcement for carbon monoxide reduction: within-subject effects of pay amount. J Appl Behav Anal 1984;17:477-83.

32 Stitzer ML, Bigelow GE. Contingent reinforcement for reduced carbon monoxide levels in cigarette smokers. Addict Behav 1982;7:403-12.

33 Volpp KGet al. A randomized controlled trial of financial incentives for smoking cessation. Cancer Epidemiology Biomarkers \& Prevention 2006;15:12-18.

34 Higgins ST, Washio Y, Heil SH, et al. Financial incentives for smoking cessation among pregnant and newly postpartum women. Prev Med 2012;55 Suppl:S33-40.

35 Dunn KE, Sigmon SC, Reimann EF, et al. A contingencymanagement intervention to promote initial smoking cessation among opioid-maintained patients. Exp Clin Psychopharmacol 2010;18:37-50.

36 Rojewski AM, Fucito LM, Baker NL, et al. A preoperative contingency management intervention for smoking abstinence in cancer patients: a preliminary randomized controlled trial. Nicotine Tob Res 2021;23:1064-7.

37 Chan A-W, Tetzlaff JM, Gøtzsche PC, et al. Spirit 2013 explanation and elaboration: guidance for protocols of clinical trials. BMJ 2013;346:e7586.

38 Benowitz NL, Bernert JT, Foulds J, et al. Biochemical verification of tobacco use and abstinence: 2019 update. Nicotine Tob Res 2020;22:1086-97.

39 Fiore M, Jaén C, Baker T. Treating tobacco use and dependence 2008 update: clinical practice guideline. Rockville, MD: USDHHS, 2008.

40 Martino S, Ball SA, Nich C, et al. Community program therapist adherence and competence in motivational enhancement therapy. Drug Alcohol Depend 2008;96:37-48.

41 Carroll KM, Nich C, Sifry RL, et al. A general system for evaluating therapist adherence and competence in psychotherapy research in the addictions. Drug Alcohol Depend 2000;57:225-38.

42 Toll BA, Martino S, O'Malley SS, et al. A randomized trial for hazardous drinking and smoking cessation for callers to a Quitline. J Consult Clin Psychol 2015;83:445-54.

43 Toll BA, O'Malley SS, Katulak NA, et al. Comparing gain- and lossframed messages for smoking cessation with sustained-release bupropion: a randomized controlled trial. Psychol Addict Behav 2007;21:534-44.

44 Toll BA, Martino S, Latimer A, et al. Randomized trial: Quitline specialist training in gain-framed vs standard-care messages for smoking cessation. J Natl Cancer Inst 2010;102:96-106.

45 Heatherton TF, Kozlowski LT, Frecker RC, et al. Measuring the heaviness of smoking: using self-reported time to the first cigarette of the day and number of cigarettes smoked per day. $\mathrm{Br} J$ Addict 1989;84:791-800.

46 Heatherton TF, Kozlowski LT, Frecker RC, et al. The Fagerström test for nicotine dependence: a revision of the Fagerström tolerance questionnaire. Br J Addict 1991;86:1119-27.

47 Kozlowski LT, Porter CQ, Orleans CT, et al. Predicting smoking cessation with self-reported measures of nicotine dependence: FTQ, FTND, and Hsi. Drug Alcohol Depend 1994;34:211-6.

48 Sobell L, Sobell M. Alcohol consumption measures. In: Allen J, Wilson V, eds. Assessing alcohol problems: a guide for clinicians and researchers, Second Edition. Bethesda, MD: NIAAA, 2003: 75-99.

49 Toll BA, Cooney NL, McKee SA, et al. Do daily interactive voice response reports of smoking behavior correspond with retrospective reports? Psychol Addict Behav 2005;19:291-5.

50 Brown RA, Burgess ES, Sales SD, et al. Reliability and validity of a smoking timeline follow-back interview. Psychology of Addictive Behaviors 1998;12:101-12.

51 Levine J, Schooler NR. SAFTEE: a technique for the systematic assessment of side effects in clinical trials. Psychopharmacol Bull 1986;22:343-81.

52 Toll BA, McKee SA, Martin DJ, et al. Factor structure and validity of the medication adherence questionnaire (MAQ) with cigarette smokers trying to quit. Nicotine Tob Res 2007;9:597-605. 
53 Snaith RP, Hamilton M, Morley S, et al. A scale for the assessment of hedonic tone the Snaith-Hamilton Pleasure scale. Br J Psychiatry 1995; 167:99-103.

54 Cappelleri JC, Bushmakin AG, Baker CL, et al. Confirmatory factor analyses and reliability of the modified cigarette evaluation questionnaire. Addict Behav 2007;32:912-23.

55 Löwe B, Wahl I, Rose M, et al. A 4-item measure of depression and anxiety: validation and standardization of the patient health Questionnaire-4 (PHQ-4) in the general population. J Affect Disord 2010;122:86-95.

56 Yong H-H, Borland R, Siahpush M. Quitting-related beliefs, intentions, and motivations of older smokers in four countries: findings from the International tobacco control policy evaluation survey. Addict Behav 2005;30:777-88.

57 Giardina TD, Hyland A, Bauer UE, et al. Which population-based interventions would motivate smokers to think seriously about stopping smoking? Am J Health Promot 2004;18:405-8.

58 Borland $\mathrm{R}$, Yong $\mathrm{H}-\mathrm{H}$, O'Connor RJ, et al. The reliability and predictive validity of the Heaviness of smoking index and its two components: findings from the International tobacco control four country study. Nicotine Tob Res 2010;12 Suppl:S45-50.

59 The WHOQOL group. Development of the world Health organization WHOQOL-BREF quality of life assessment. The WHOQOL group. Psychol Med 1998;28:551-8.

60 Browne RH. On the use of a pilot sample for sample size determination. Stat Med 1995;14:1933-40.

61 Julious SA, Owen RJ. Sample size calculations for clinical studies allowing for uncertainty about the variance. Pharm Stat 2006:5:29-37.
62 Ellenberg JH. Intent-To-Treat analysis versus As-Treated analysis. Drug Inf J 1996;30:535-44.

63 Liu Y, De A. Multiple imputation by fully conditional specification for dealing with missing data in a large epidemiologic study. Int J Stat Med Res 2015;4:287-95.

64 von Hippel PT. How many Imputations do you need? A twostage calculation using a quadratic rule. Sociol Methods Res 2020;49:699-718.

65 Kraemer HC, Wilson GT, Fairburn CG, et al. Mediators and moderators of treatment effects in randomized clinical trials. Arch Gen Psychiatry 2002;59:877-83.

66 Smith PH, Bessette AJ, Weinberger AH, et al. Sex/gender differences in smoking cessation: a review. Prev Med 2016;92:135-40.

67 Kulak JA, Cornelius ME, Fong GT, et al. Differences in quit attempts and cigarette smoking abstinence between whites and African Americans in the United States: literature review and results from the International tobacco control us survey. Nicotine Tob Res 2016;18 Suppl 1:S79-87.

68 U.S. Department of Health and Human Services. Tobacco use among U.S. racial/ethnic minority Groups - African Americans, American Indians and Alaska natives, Asian Americans and Pacific Islanders, and Hispanics: a report of the surgeon General. In: services $\mathrm{DoHaH}$, ed. Atlanta, Georgia: Centers for Disease Control and Prevention, National Center for Chronic Disease Prevention and Health Promotion, Office on Smoking and Health, 1998.

69 Weinstein MC, Siegel JE, Gold MR, et al. Recommendations of the panel on cost-effectiveness in health and medicine. JAMA 1996;276:1253-8.

70 SAS. Cary, NC: SAS Institute Inc, 2013. 